\title{
3D Spectroscopy with large Telescopes: past, present and prospects
}

\author{
G. Monnet
}

Observatoire de Lyon, 69561 Saint-Genis-Laval Cedex, France

\begin{abstract}
The dimensional curse of having to pack 3D spectrophotometric data on solely 2D detectors is briefly discussed, and the main astronomical domains covered by 3D spectroscopy introduced. Finally, the scientific case for high spatial resolution 3D techniques coupled to Adaptive Optics capabilities is presented, with special emphasis on the observation of the nuclei of galaxies.
\end{abstract}

\section{Introduction}

Full kinematical investigation of any extended astronomical object, e.g. a given stellar population in a galaxy or an ionized gas (HII) region, would in principle require to obtain its distribution function. This is a function in the 6-D phase space, with 3 space and 3 velocity cocrdinates. Is is of course well known that, apart for a few nearby objects, only three of these coordinates are experimentally accessible: the two spatial coordinates in the plane of the sky and the radial component of the velocity. Hence the general name of $3 \mathrm{D}$ spectroscopy for all techniques which give such "data cubes" and thus are able to extract all available spectrophotometric information on astronomical objects for kinematical as well as physical studies.

\section{The dimensional curse}

Present detectors are unfortunately of a two-dimensional nature only (see, however, an analysis of potential 3D detectors by P. Connes during the Colloquium). This makes it a major problem to pack the inherently 3D spectrophotometric information content $(x ; y ; \lambda)$ of celestial objects on the detector chip. This, in turn, explains the still much prevalent use of classical long-slit spectrographs, despite total loss of one of the spatial coordinates (across the slit width) and because of their perfect match with available digital detectors.

There are two general classes of solution to this problem: spectrometers, for which time scanning is used to provide the missing third dimension, and integral field spectrographs (IFS) which manage to cram the full data from a single exposure on the chip. Various examples of the first class, e.g. the $\lambda$-scanning Fabry-Perot (SFP) or Fourier transform spectrometers and the y-scanning longslit grating spectrometer have been presented during the Colloquium. For the second class, both the fiber and the lenslet arrays solutions have also been heavily discussed. 


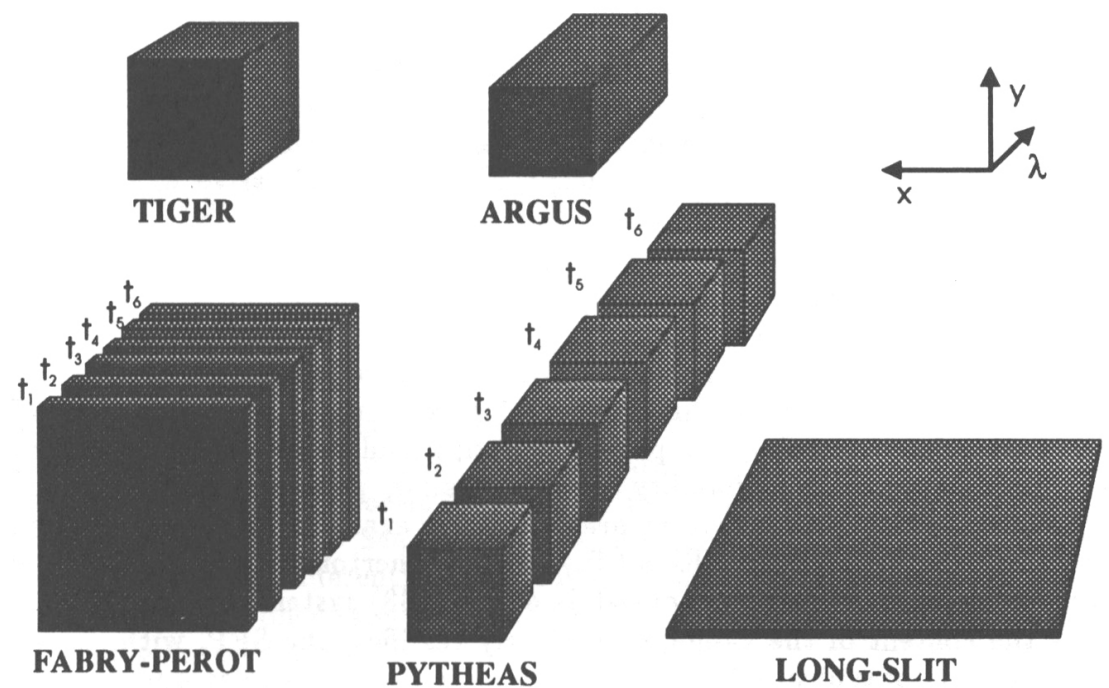

Figure 1. The spatio-spectral geometry of various 3D spectrophotometric systems (TIGER, ARGUS, FABRY-PEROT, PYTHEAS) is shown, with the 2D (LONG-SLIT) spectrograph for comparison. ( $x$, $y)$ : spatial coordinates. $\lambda$ : wavelength. $t$ : time.

Figure 1 shows the geometry in $x ; y ; \lambda$ coordinates of some of the major 3D spectrophotometric systems developed so far. The 2D classical long-slit spectrograph has been added as a comparison. Note that for spectrometers, the number of successive exposures is generally limited to well below a hundred, while for spectrographs the spatial field can hardly be greater than, say, $40 \times 40$ pixels because of harsh competition between spatial and spectral pixels for the CCD total available area. This for instance explains why, in figure 1 , a given SFP exposure (FABRY-PEROT) appears as a slab, while the IFSs (TIGER and ARGUS) are beam-shaped. One can also see the PYTHEAS system (see E. Lecoarer 1994, these proceedings), an hybrid system with the relatively large wavelength range ( $\geq 500 \AA$ ) of its IFS-type basic design, but with a spectral resolution boosted by about a factor 10 with the addition of a scanning FabryPerot, acting as a tunable filter.

\section{The scanning Fabry-Perot Spectrometer}

The ancestor of all 3D spectroscopic devices came into existence more than 80 years ago and a few kilometers only from the Colloquium location, when Buisson, Fabry and Bourget turned a Fabry-Perot etalon on the Orion nebula, obtaining quite detailed informations on its ionized gas content (Buisson et al. 1914). This technique was revived in the early 50's, again at the Observatoire de Marseille, by G. Courtès, and further work by his and other groups led to 
extensive kinematical studies of HII regions in our Galaxy and in nearby, gasrich, external galaxies (see e.g. Carranza et al. 1968, Courtès 1972).

This technique was not however truly 3-dimensional, as only the regions which emit an emission line that fortuitously happens to be transmitted by the etalon are observed in a given exposure. It was B. Tully, then at the University of Maryland, who discovered the concept of making successive exposures at different separation of the etalon plates (up, when needed, to a full scanning of its free spectral range), and using them to reconstruct an (emission) line profile at each point of the field. This led to the first spectrophotometric data "cube", on the galaxy M51 (Tully 1974).

Further development, from Tully's prototype to the modern SFP spectrometer was made possible by two major technological achievements: a) the advent of 2D digital detectors, first the photon-counting and then the CCD cameras, and b) the commercial availability of high quality, piezoelectrically scanned, Fabry-Perots from Queensgate Corporation. Early examples of this new class of instruments are the RGO TAURUS (Taylor and Atherton 1980) and the Observatoire de Marseille CIGALE (Boulesteix et al. 1983) systems.

As the content of the Colloquium clearly testifies, the SFP, with its relatively large field of typically $10 \times 10$ arcmin. for a $4 \mathrm{~m}$. class telescope, has been widely used for kinematical studies of extended ionized gas regions, ranging from the Io torus to distant radiogalaxies, but with special emphasis to detailed radial velocity maps on relatively nearby galaxies. As noted above, one practical limitation of this technique is the small number (rarely above 50) of spectral "pixels". This makes the study of line ratios quite difficult, with the need of photometrically calibrated objects in the field. Furthemore, the instrument is badly adapted to absorption line studies of even a single line, again because a very careful spectrophotometric calibration of the interference filter used as an order sorting device, would be needed. Practically speaking, the only reachable objects in that field appear to be Galactic globular clusters, which velocity dispersions are low enough to require scanning by a small fraction of an order, and where indeed successful observations with an SFP have recently been achieved by T. Pryor of Rutgers University.

The first 3D device able to fully study the stellar populations of an external galaxy has been the scanning long-slit grating spectrograph developed by Wilkinson et al. 1986. Used as a spectrometer and from 71 successive exposures with 2.4 arcsec. consecutive offsets on the sky, it gave a complete radial velocity map of the mean stellar motions in the $120 \times 200$ arcsec. central region of Cen $A$ (NGC 5128). As for all spectrometric techniques, there is however a corresponding penalty in sensitivity: this is especially painful for absorption line studies, for which even the central part of such bright galaxies provide relatively meager photon fluxes. Coupled with the (quasi) absolute need for a photon-counting detector at a time where most observatories were turning towards CCDs, this probably explains why this technique has been seldom used and why the socalled integral field spectrographic techniques have been developed, essentially to provide the same kind of data, and despite their significantly smaller sky coverage. 


\section{Integral Field Spectrography: the case for high spatial resolution}

The first such system, invented by Courtès 1982, was basically a multi-slit spectrograph, where the slitlets are replaced by small multiple images of the pupil of the telescope given by a lenslet array, illuminated by a much enlarged image of the field (see e.g. R. Bacon 1994, these proceedings). Another version (Vanderriest et al. 1984) used a modified long-slit spectrograph, where the slit is replaced by a tapered fiber bundle (see e.g. C. Vanderriest 1994, these proceedings).

Both systems, presently in use at various large telescopes, permit a full 3D coverage in their small, typically $5 \times 5$ arcsec., fields. As such, they are clearly optimized for the study of compact structured objects with complex photometric and/or physical properties. This makes for a large scientific range, going from the geology of telluric planets to the environment of quasars and encompassing both quiet and active galactic nuclei as prime targets, as amply demonstrated during the Colloquium. See for instance the complex structure of the central part of M 31 with differently offsetted photometric and dynamical "centers", or the multi-component stellar population content of that of M 104 (Emsellem 1994, these proceedings).

One of the main interest of these IFS techniques is to provide continuum and line maps directly derived from the data cube. As shown e.g. by Bacon et al. 1994 in their detailed study of the nucleus of M31, it can be used: a) to pinpoint spectral details to better than 0.1 arcsec. relative spatial accuracy, and $b$ ) to derive the spatial point spread function of the spectrographic observations for proper model testing and even for (partial) deconvolution of the spectrographic data. This, in turn, makes them highly attractive for proper use of the sub-0.1 arcsec. images that will soon be delivered through adaptive optics corrections at most large telescopes. Figure 2, based on a very recent HST image of the canonical Seyfert galaxy NGC 1068, illustrates the very poor coverage of conventional long-slit spectrography, as compared with IFS. This can be somewhat alleviated by opening the slit, but at the expense of not only a loss in spectral resolution, but also of significant spectral distorsions of the data (the "slit" effect). The potential scientific range of such observations is quite large, and again includes the study of both quiet and active nuclei of nearby galaxies as major examples, e.g. for the quest for central supermassive black holes as the engine responsible for nuclear activity.

\section{Conclusion}

Both multi-slit and multi-fiber spectrographs are presently widely used at every large telescope in operation for the study of diluted objects, e.g. star clusters or galaxies in distant Clusters. Moreover, they are systematically planned for all of the $8-10 \mathrm{~m}$. class projects, as general use instruments. Contrarily, 3D spectrometers as well as 3D spectrographs are still generally operated by specialized teams and, at least so far, play a small role (if any) for this new instrumentation. This is in sharp contrast with the relative extent of their (largely disjointed) respective scientific domains, for which 3D instruments compare quite favourably, as well as for new needs arising from the widespread appearance of adaptive optics capabilities at large telescopes. The reasons for this neglect are quite complex 


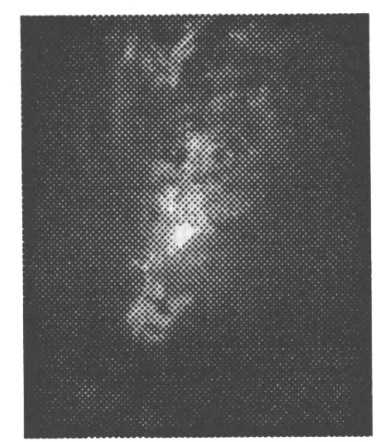

NGC 1068

HST FOC + COSTAR observation Machetto et al.

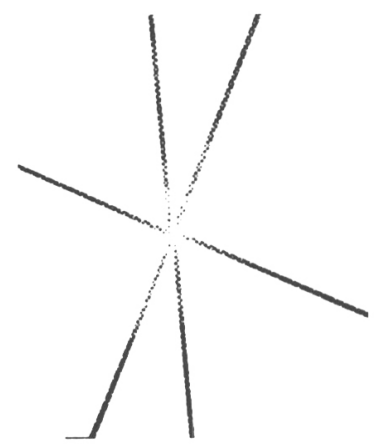

Three long-slit observations

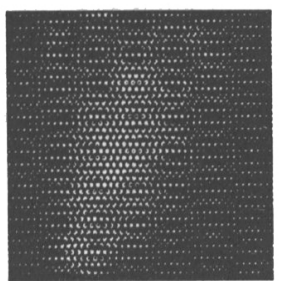

One IFS observation

Figure 2. Central region of NGC 1068. Left: 7" $\times 6$ " HST Image in the OIII line. Center: Potential coverage from 3 long-slit exposures. Right: Potential coverage from a single IFS exposure with 1600 "spatial" pixels. All three images are shown at the same orientation and scale.

and may involve some conservatism, but one stands out clearly: the general lack of efficient, robust, portable, user-friendly, well documented and maintained, processing softwares for proper reduction of the data cubes provided by these instruments by a non "expert" in the field.

\section{Discussion}

J. Bland-Hawthorn: Your TIGER observations of M 31 and M 104 are nothing short of remarkable. Which wavelength were used and what signal-to-noise ratio do you reach?

G. Monnet: We used the classical $5200 \AA$ to $5700 \AA$ wavelength range with offers the $\mathrm{MgI}$ triplet and a number of metallic blends. A minimum signal-tonoise ratio of about 30 is needed to derive accurate enough mean velocities and velocity dispersion fields.

\section{References}

Bacon, R., Emsellem, E., Monnet G., Nieto J.L. 1994, A\&A, 281, 691

Boulesteix, J., Georgelin, Y., Marcelin, M., Monnet, G., in S.P.I.E. Conf. Inst. Astr.: 1983, vol. V, 445

H., Buisson, Ch. Fabry, H. Bourget 1914, ApJ, 40, 241

Carranza, G., Courtès, G., Georgelin, Y., Monnet, G., Pourcelot, A. 1968, Ann. d'Ap., 31, 63 
Courtès, G. 1972, Vistas in Astron., 14, 163

Taylor, K., Atherton, P. 1980, MNRAS, 191, 675

Tully, R.B. 1974, ApJS, 27, 415

Vanderriest, C., Courtès, G., Donas, J. 1984, J. Optics (Paris), 15, $n^{\circ} 4$ bis, 237

Wilkinson, A., Sharples, R.H., Fosbury, R.A.E., Wallace, P.T. 1986, MNRAS, 218,297 